\title{
Optimización superficial de alambres de ortodoncia de Ni-Ti superelástico mediante nitruración gaseosa. Parte II: Cuantificación de la mejora de la nanodureza y el coeficiente de fricción
}

\author{
F.BOCCIO, F.J.GIL", A. MEMBRIVE* ,M.V.ALFONSO, \\ A.CAMPOS, E.SOLANO Y J.A.PLANELL \\ Grupo de investigación de Ortodoncia. \\ Facultad de Odontología. Universidad de Sevilla \\ *CREB. Dpto. Ciencia de los Materiales e Ing. Metalúrgica,. \\ Universidad Politécnica de Cataluña
}

\begin{abstract}
Resumen
La mejora del deslizamiento entre el alambre de ortodoncia y el bracket es uno de los principales problemas en la terapia ortodoncica. Este estudio presenta la nitruración gaseosa como un eficaz método de mejora del deslizamiento de los alambres ortodóncicos de Ni-Ti superelástico (composiciones cercanas a la equiatómica) respecto al bracket, principal inconveniente de estas aleaciones en la aplicación ortodóncica. Mediante microscopía óptica y electrónica de barrido se comprobó la existencia de una capa superficial nitrurada, así como la morfología de las fases presentes. Asimismo, se realizaron microanálisis químicos de las capas exteriores mediante técnica de energía dispersiva de rayos $X$. En todas las muestras nitruradas la dureza aumentó con la severidad del tratamiento y se alcanzaron valores de hasta $1111 \mathrm{HV}$ en comparación con los valores de $323 \mathrm{HV}$ del alambre sin tratar. Se diseñaron ensayos de tipo pin on disk adaptados a las peculiaridades de los alambres, que mostraron un descenso del coeficiente de fricción. El endurecimiento y mejora de la fricción en la superficie del material se justifican por la formación de las capas nitruradas.
\end{abstract}

Palabras clave: Superelasticidad, NiTi, Nitruración, Fricción.

\section{Summary}

The improvement of the slipping between the orthodontic archwire and the bracket is one of the most important problems in orthodontic therapy. In this work it is shown that the nitrogen diffusion produces an important improvement of the slipping between the superelastic NiTi orthodontic archwires (with chemical composition near the equiatomic percentage) and the bracket. The high value of the friction coefficient is the main problem for the application of the NiTi superelastic archwires. By means of optical and scanning electron microscopy the titanium nitride coating and its microstructure is shown. Besides, the $X$-ray dispersive energy is used in order to determine the nature of this coating. The hardness increases from $323 \mathrm{HV}$ to $1111 \mathrm{HV}$ with the treatment. Pin on disk tests were performed and a decrease of the friction coefficient from 0.52 to 0.28 was observed. Both the hardening and the decrease of the friction coefficient are attributed to the growth of the titanium nitride coating.

Keywords: Superelasticity, NiTi, Nitrogen diffusion treatment. 


\section{Introducción}

El material que ofrece unas propiedades más adecuadas en muchas etapas del tratamiento en su uso como arco ortodóncico es el Ni-Ti superelástico [1-3], precisamente porque su superelasticidad transformacional (en la que se induce una transformación martensítica al cargar y la retransformación al descargar) le permite aplicar unas tensiones moderadas al periodonto, sobrellevando, sin embargo, deformaciones relativamente grandes. Es pues un material con una recuperación (springback) excelente y con una muy baja rigidez. Esto es de vital importancia en ortodoncia ya que las fuerzas de baja intensidad mantenidas de manera continua son las que han demostrado una mayor efectividad y un menor período de tratamiento en la corrección de maloclusiones dentales [4-5].

Sin embargo, los arcos ortodóncicos de Ni-Ti comercializados actualmente presentan unas malas propiedades de deslizamiento respecto a su elemento de guiado. La deformación plástica superficial y la elevada fricción con el bracket puede hacer inútiles los esfuerzos de un determinado arco e incluso provocar movimientos dentarios no deseados.

Frente a las muchas alternativas de tratamientos de optimización superficial, se ha confiado en un tratamiento termoquímico superficial de nitruración gaseosa, principalmente por su sencillez y economía (sobre todo frente a la alternativa de los brackets superdeslizantes) [6-13], y el objetivo de esta parte del trabajo es estudiar la cuantificación de la mejora en la nanodureza superficial y la fricción con el bracket.

\section{Material y metodología experimental}

El material empleado fue Ni-Ti superelástico (44.1\% en peso $\mathrm{Ti}$ ) en forma de arcos de ortodoncia comerciales de sección circular (diámetro 0.018"), suministrados por la firma $\mathrm{ORMCO}^{\circledR}$, con una nanodureza media medida de $323 \mathrm{HV}$.

Por lo que respecta al método de nitruración nos referimos a [2], con los mismos niveles de las variables: dos métodos de extracción de la muestra, el primero, bajo un elevado flujo circulante de $\mathrm{N}_{2}$ para evitar la oxidación, e inmediatamente temple en agua a $20^{\circ} \mathrm{C}$; el segundo, después de un enfriamiento en horno, siempre en atmósfera de $\mathrm{N}_{2}$, circulando el flujo imprescindible para evitar la oxidación; tres temperaturas de nitruración, 800,
900 y $1000^{\circ} \mathrm{C}$ (superiores a la de recristalización, situada alrededor de $700^{\circ} \mathrm{C}$ ) tanto para las muestras templadas como para las dejadas enfriar en el horno; tiempos de nitruración de 15, 30, 45 y 60 minutos para las muestras templadas en agua y de $3,5,10$ y 20 minutos para las muestras dejadas enfriar en horno.

Las muestras para observación al microscopio óptico y electrónico se prepararon embaquelitando los alambres, puliéndolos y atacando con una solución $30 \% \mathrm{HNO}_{3}, 24 \% \mathrm{HF}$ y el resto agua destilada. Además, en la muestra para observación al microscopio electrónico se tuvo que dar un recubrimiento de oro.

Se midió la nanodureza con un equipo Fisher empleando cargas de $1 \mathrm{~g}$ durante $15 \mathrm{~s}$; las huellas se midieron con un microscopio óptico Olympus acoplado al equipo de dureza.

De aquellas muestras por enfriamiento en horno se realizaron ensayos de coeficiente de fricción en función de la distancia deslizada. La filosofía de este ensayo fue lo que podría llamarse wire on disk por su analogía con el tipo pin on disk. El alambre nitrurado se fijó cuidadosamente con adhesivo de cianoacrilato en un portamuestras de baquelita sin que el adhesivo contactara la superficie que el alambre ofrecía al disco de desgaste. Se emplearon dos discos distintos de desgaste:

- Disco (1): Material, Ti-6Al-4V; microestructura "mill annealed"; microdureza 353HV.

- Disco (2): Material, acero inoxidable austenítico AISI 316; microestructura austenítica; microdureza $453 \mathrm{HV}$.

El contacto del alambre con el disco fue plano y en el sentido longitudinal, a fin de simular al máximo el contacto arco-bracket. Se empleó una velocidad angular de los discos relativamente baja de $0,5236 \mathrm{rad} / \mathrm{s}$, ofreciendo al alambre distintos diámetros de rodadura para evitar la fricción con zonas de superficie previamente desgastadas. Idealmente, la carga normal sobre el alambre debería haber sido de $1 \mathrm{~N}(98,1 \mathrm{~g}$ fuerza) a fin de simular la carga en servicio (en las que valores típicos van desde 20 a 100g fuerza). Sin embargo, se emplearon $10 \mathrm{~N}$ para evitar que cualquier desviación sobre el ideal plano de contacto afectara significativamente al coeficiente de fricción. En efecto, se comprobó que una pequeña desviación del plano ideal de contacto proporcionaba un error del orden de las décimas de newton en la lectura de la fuerza tangencial, con lo que el coeficiente de 
fricción se hubiera visto seriamente alterado, ya que éste resulta del cociente entre esfuerzo tangencial y normal.

\section{Resultados y discusión}

La observación al microscopio óptico reveló la presencia de placas de martensita en el material en estado de recepción. Previsiblemente, entre la martensita se encuentran granos de fase $\beta$, ya que a temperatura ambiente, la de la observación, se sabe que coexisten ambas fases [2]. Sin embargo, es posible que en la preparación de muestras se haya inducido por tensión placas de martensita superficiales en el proceso de pulido. Este fenómeno parece observarse también en las muestras de material tratado, que presentan una estructura aparentemente martensítica. En la Figura 1 puede verse la imagen al microscopio óptico de una muestra nitrurada a $1000^{\circ} \mathrm{C}$ durante 20 minutos. Se puede apreciar la presencia de una capa superficial resultado de la nitruración, que gracias al microscopio electrónico puede apreciarse mejor (véase Figura 2).

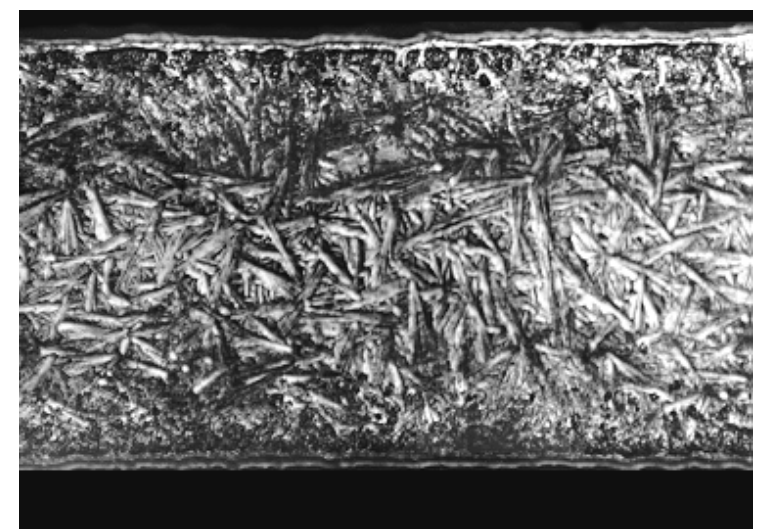

Figura 1. Alambre de ortodoncia tratado a $1000^{\circ}$ durante 20 minutos (x500).

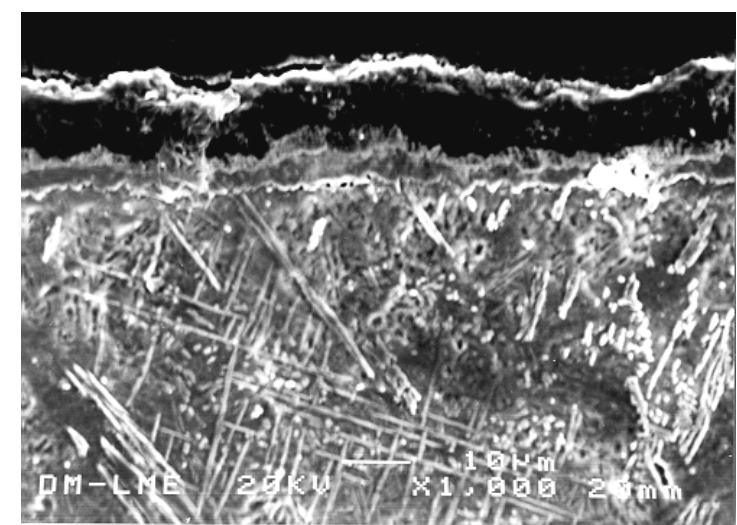

Figura 2. Capa nitrurada en el alambre de la figura anterior (x1000)
Si se observa con detalle, puede apreciarse la existencia de dos subcapas: una más amplia y oscura en el exterior y otra más delgada en la parte interior.

Microanálisis por técnica de energía dispersiva de rayos $\mathrm{X}$ revelaron que la subcapa exterior está seguramente compuesta en su mayor parte por óxidos y oxinitruros de titanio, y que la subcapa interior probablemente contiene mayoritariamente nitruros de titanio.

Los valores de las nanodurezas mostraron un claro aumento del valor Vickers a medida que tiempos y temperaturas de nitruración se hacen mayores, tanto en las muestras templadas, (Figura 3), como las dejadas enfriar en horno, (Figura 4).

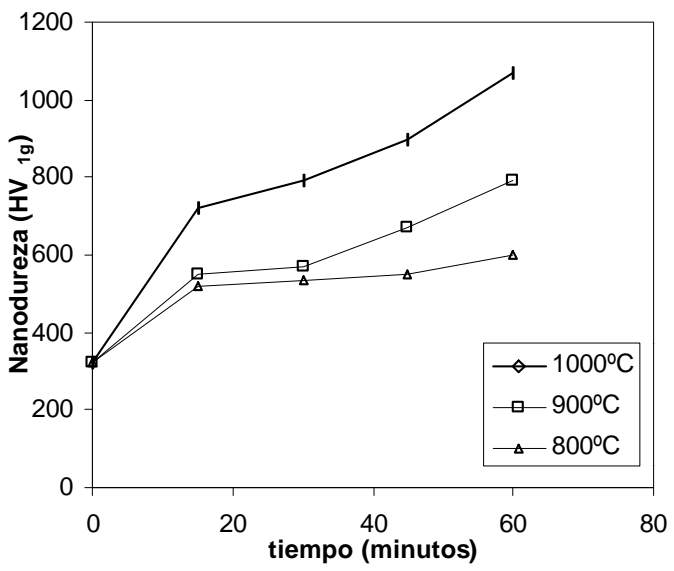

Figura 3. Evolución de la nanodureza con el tiempo y la temperatura de tratamiento para las muestras templadas en agua

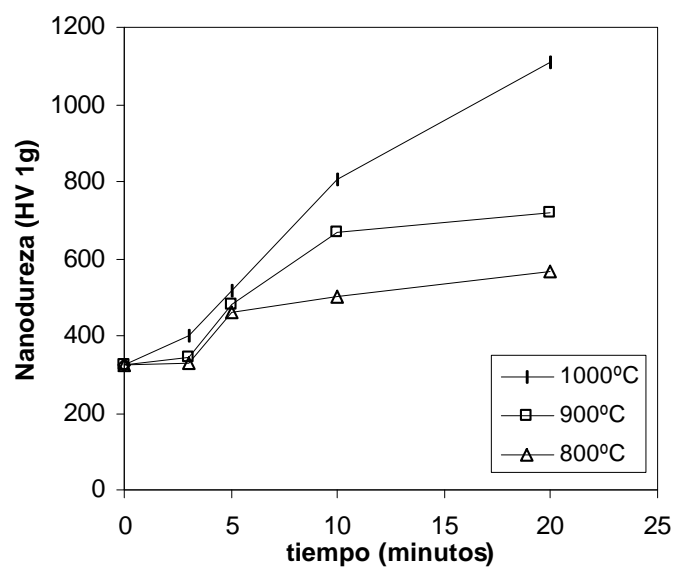

Figura 4. Evolución de la nanodureza con el tiempo y la temperatura de tratamiento para las muestras dejadas enfriar en horno.

Obsérvese que la dureza del material en estado de recepción es de 323 HV y que en el caso más extremo se alcanza $1111 \mathrm{HV}$. Tras nitrurar las muestras, se observó en todos los casos el color 
amarillo característico de los nitruros de titanio, nitruros delta, y oxinitruros, lo que hace pensar que la matriz se empobreció en Ti. Dicha capa de compuestos es extremadamente delgada y dura.

En los casos en que se constató una cierta oxidación se detectó fragilidad de dicha capa, que se corresponde sin duda a la subcapa exterior observada por microscopía, a modo de cascarilla que se desprende rápidamente en la fricción. Obsérvese que, a mismas condiciones interpolables de nitruración, las muestras enfriadas en horno presentan durezas mayores respecto a las templadas en agua. Esto es debido principalmente al mayor tiempo de permanencia de las muestras enfriándose desde altas temperaturas en atmósfera de nitrógeno.

Los puntos obtenidos en los ensayos de desgaste se ajustaron en todos los casos a una curva polinómica de grado 6 por el método de los mínimos cuadrados.

Para el disco (1), se observó en prácticamente todos los casos (a excepción de la muestra sin tratar y del caso $3^{\prime} 800^{\circ} \mathrm{C}$, precisamente el tratamiento menos severo) una zona de aumento del coeficiente de fricción correspondiente a las capas más exteriores y la estabilización posterior alrededor del valor que presenta el material en estado de recepción (Figura 5). En los casos en los que el tratamiento es más severo, la zona de bajo coeficiente de fricción es más amplia debido a una mayor profundidad de alcance del nitrógeno intersticial. Los valores del coeficiente de fricción para bajas distancias en estos tratamientos son muy aceptables (alrededor de 0,28) frente al material en estado de recepción (alrededor de 0,5). Estos resultados confirman las expectativas fundadas en los espectaculares aumentos de nanodureza. Se podría objetar que la zona con bajos coeficientes de fricción es en todos los casos muy estrecha, pero en el caso que nos ocupa ya es suficiente porque se trata de un problema de deslizamiento que se relaciona más bien con un coeficiente de fricción estático y no tan sujeto a las variaciones por desgaste que con un coeficiente de fricción dinámico. De hecho, en estos ensayos se mide un coeficiente de fricción dinámico (a pesar que la velocidad se ha escogido lo más baja posible) pero éste ya nos puede dar una idea, sobre todo para bajas distancias de desgaste, del coeficiente de fricción estático. Además, teniendo en cuenta que no podemos alterar la superelasticidad en un alambre de tan poca sección, nos interesa que la capa de endurecimiento sea lo más pequeña posible.

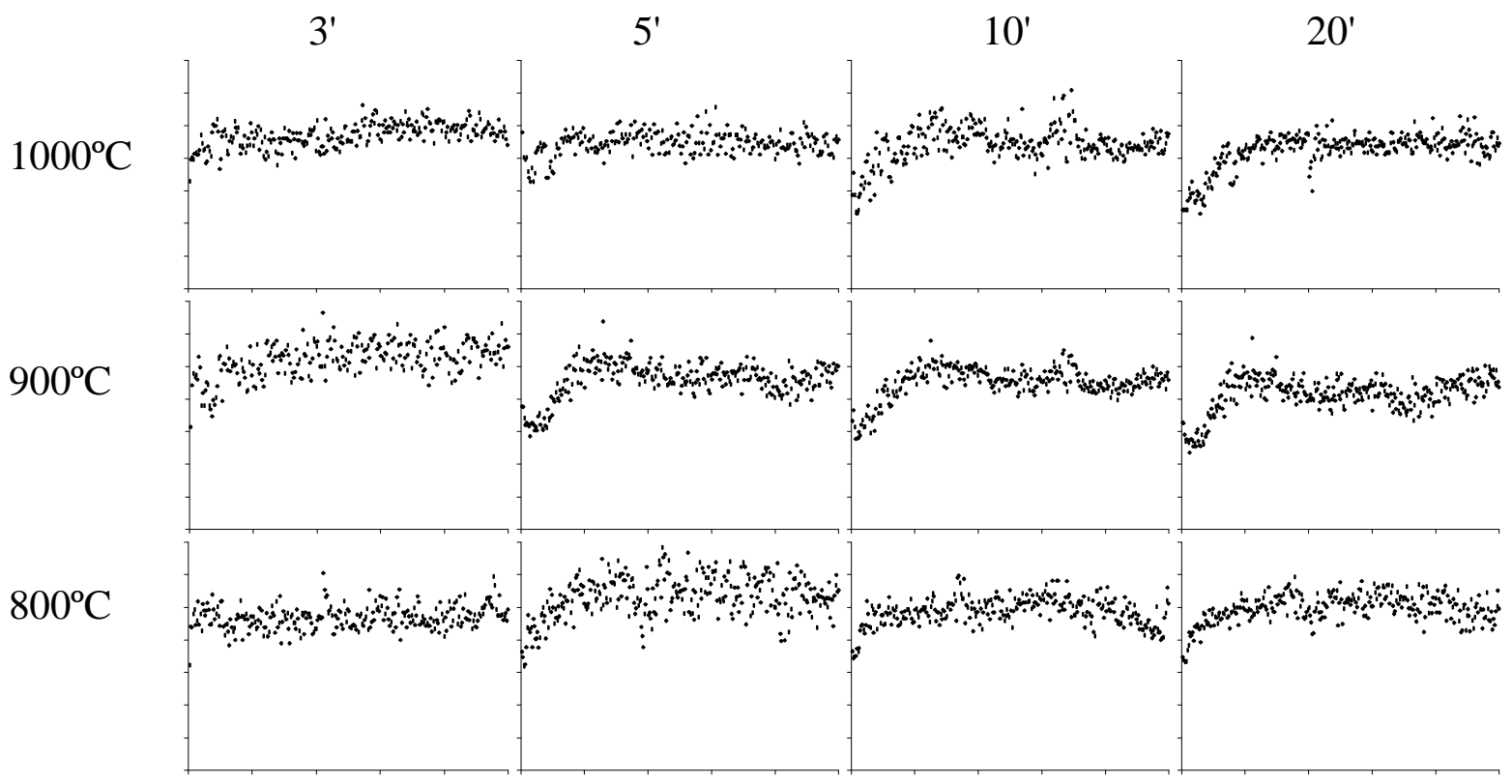

Figura 5. Coeficientes de fricción en función de la distancia deslizada contra disco (1). Las divisiones representan 1 décima y $10 \mathrm{~m}$ en los ejes vertical y horizontal respectivamente. 
En la Figura 6 se toman los valores intrapolados de los coeficientes de fricción para una distancia recorrida de desgaste de $1 \mathrm{~m}$ contra el disco (1), en función del tratamiento, de manera que se puede observar claramente el descenso del coeficiente de fricción con la severidad del tratamiento.

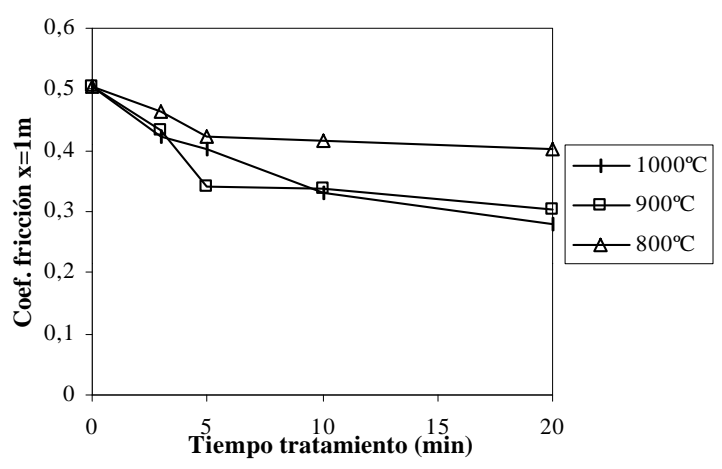

Figura 6. Coeficientes de fricción interpolados (a partir de la regresión polinómica de grado seis) para una distancia recorrida de desgaste de $1 \mathrm{~m}$ contra disco (1) en función del tratamiento.
Hay que tener en cuenta que un tiempo nulo de tratamiento corresponde a los valores del material en estado de recepción.

Los coeficientes de fricción contra el disco (2), (Figura 7) apuntan unos resultados hacia la misma dirección que el anterior, aunque no tan claramente en el mayoría de los tratamientos. Solamente en los tratamientos más severos se observa un coeficiente de fricción claramente menor en la capa exterior del material, que llega a ser de 0.32 respecto a 0.52 para las muestras sin tratar. La Figura 8 recoge los valores interpolados de los coeficientes de fricción para una distancia recorrida de desgaste de $1 \mathrm{~m}$ contra el disco (2), en función del tratamiento.

Es notable destacar la correlación de los valores de coeficiente de fricción con las nanodurezas obtenidas, de manera independiente al tiempo y temperatura del tratamiento, (Figura 9 y Figura 10). Ocurre que a más nanodureza menos coeficiente de fricción (en una relación que parece lineal), pero esto no tiene que ser siempre cierto debido, en general, a la fragilidad de capas muy duras.

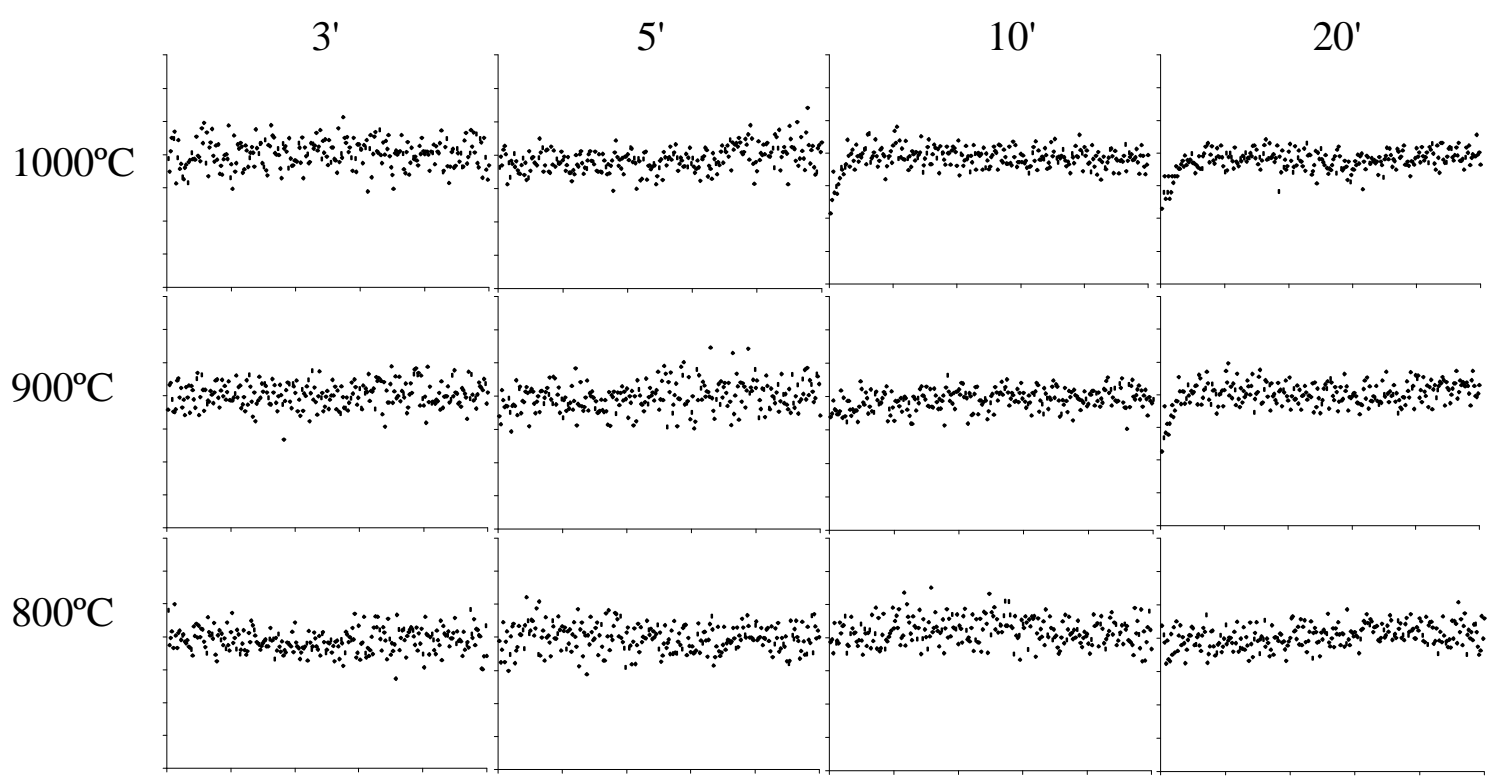

Figura 7. Coeficientes de fricción en función de la distancia deslizada contra disco (2). Las divisiones representan 1 décima y $10 \mathrm{~m}$ en los ejes vertical y horizontal respectivamente 


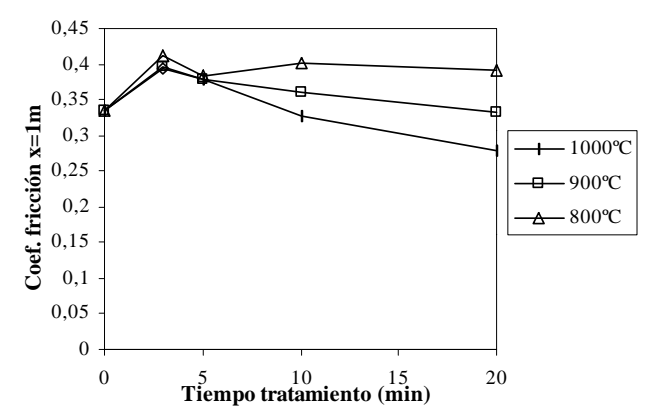

Figura 8. Coeficientes de fricción interpolados (a partir de la regresión polinómica de grado seis) para una distancia recorrida de desgaste de $1 \mathrm{~m}$ contra disco (2) en función del tratamiento

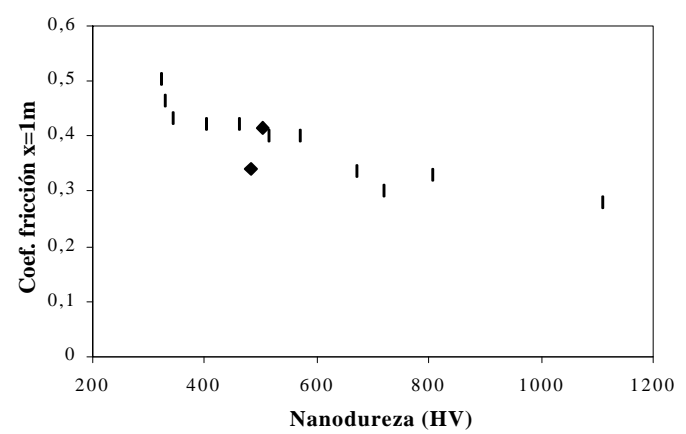

Figura 9. Correlación entre nanodureza y coeficiente de fricción interpolado para una distancia recorrida de desgaste de $1 \mathrm{~m}$ contra $\operatorname{disco}(1)$

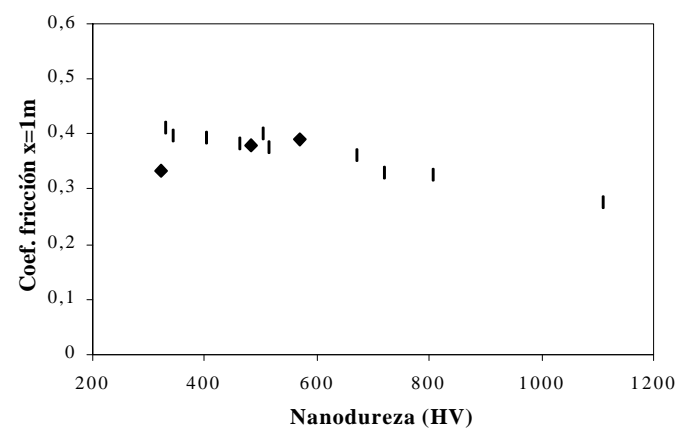

Figura 10. Correlación entre nanodureza y coeficiente de fricción interpolado para una distancia recorrida de desgaste de $1 \mathrm{~m}$ contra disco (1)

\section{Conclusiones}

El método de nitruración permite lograr un acusado endurecimiento que se forma gracias a la difusión gaseosa. Favorecida por las altas temperaturas, la difusión facilita la incorporación de nitrógeno intersticial. Los pequeños átomos de nitrógeno introducidos entre la red de la matriz contribuyen al endurecimiento avalado por las mediciones. El otro elemento contribuyente es la la capa exterior de compuestos formada; la subcapa interior observada es seguramente la más decisiva, ya que los nitruros de titanio tienen durezas por sí solos de hasta $1500 \mathrm{HV}$; la subcapa de oxidación, aunque confiere dureza, no es deseable desde el punto de vista de esta aplicación ortodóncica.

En las muestras dejadas enfriar en horno, las nanodurezas alcanzaron valores de hasta $1111 \mathrm{HV}$ en comparación con los valores de $323 \mathrm{HV}$ del alambre sin tratar. Se puede endurecer de manera suficiente sin tener que llegar a un extremo tal que fragilice la superficie del material, de manera que se mejoren de manera ostensible las propiedades de deslizamiento arco-bracket.

Los ensayos de desgaste confirman las expectativas creadas por valores tan elevados de nanodreza, observándose coeficientes de fricción más bajos en las capas más exteriores (la capa afectada por los nitruros y el nitrógeno intersticial no es demasiado profunda) a medida que aumenta la severidad del tratamiento.

Otros trabajos han estudiado cómo la nitruración ha afectado a las propiedades comentadas que hacen del Ni-Ti superelástico un material ideal para ser utilizado como arco de ortodoncia, tanto por lo que respecta a la pérdida de propiedades superelásticas como a la liberación de iones al medio salivar. Visto el conjunto de resultados, puede decirse que la sencillez, economía y las altas nanodurezas que permiten unos bajos coeficientes de fricción estáticos, hacen del método de nitruración gaseosa un candidato prometedor en su aplicación ortodóncica.

\section{Bibliografía}

1. Tosho S. Shape Memory Alloys. Volume 1. Ed. H. Funakubo. Gordon and Breach Science Publishers. Tokio. p.23. (1984)

2. Perkins. J. "Shape Memory effects in alloys". Plenum Press. New York. p. 12 (1975).

3. Iwasaki K., Hasiguti RS. "Martensitic Transformation". Lovaine: The Institute of Metals, 198. (1982)

4. Guillemany JM, Gil FJ, Miguel JR. "Ciencia y Tecnología de materiales con memoria de forma: Propiedades y Aplicaciones. Rev. Técnica Metalúrgica. 5, 213-224 (1986)

5. D.R. Hawkings en Metals Handbook, Vol. 8, American Society for Metals, 8va edición, New York (1973).

6. P. Moine, O. Popoola y J.P. Villain. Scr. Metall, vol. 20, p. 305, (1986). 
7. D.A. Porter y K.E. Easterling, Phase Transformations in Metals and Alloys, Chapman \& Halls, London (1991).

8. J.M. Prado, A. Herrero, J.A. Planell y J. Tartera, Metalúrgia General. Volumen 1, Depto. Ciencia de los Materiales e Ing. Metalúrgica, Barcelona (1994).

9. J.M. Prado, A. Herrero, J.A. Planell y J. Tartera, Metalúrgia General. Volumen 2, Depto. Ciencia de los Materiales e Ing. Metalúrgica, Barcelona (1994)

10. Shetty RH, Method of surface hardening orthopedic implant devices, US Patent n. 5.326.362,
Jul. 5, 1994.

11. Shetty RH, Mechanical and corrosion properties of nitrogen diffusion hardened Ti-6Al-4V alloy, Medical applications of ti and its alloys, ASM-STP 1272, ISBN 0-8031-2010-9 (1996).

12. Sioshansi $\mathbf{P}$, Improving the properties of titanium alloys by ion implantation, JOM, 3, 30-31 (1990).

13. Streicher RM, Semlitsch $M$, Schön R, Weber $H$, Rieker C, Metal-on-metal articulation for artificial hip joints: laboratory study and clinical results, Proc Instn Mech Engrs Part H, 210, 223-232 (1996). 